\title{
ON THE MICROLOCAL CUT-OFF OF SHEAVES
}

\author{
Andrea D'Agnolo
}

\section{Introduction}

A fundamental tool in the microlocal study of sheaves (as developed in [5]) is the so-called "microlocal cut-off lemma". More precisely, let $X$ be a $C^{\infty}$ manifold, let $p \in \dot{T}^{*} X$, set $x_{\circ}=\pi(p) \in X$, and let $\gamma$ be a proper convex conic open neighborhood of $p$ in $T_{x_{\circ}}^{*} X$. The microlocal cut-off lemma [5, Proposition 6.1.4] gives a functorial way of associating with a sheaf $F$ on $X$ another sheaf $F^{\prime}$ which is isomorphic to $F$ in $\gamma$, and whose micro-support over $x_{\circ}$ is contained in $\bar{\gamma}$ and close to that of $F$.

It turns out that the above mentioned result is not sufficient for many applications. In particular, the hypothesis of $\gamma$ being convex and proper is sometimes too strong. The aim of this paper is to state a refined version of the microlocal cut-off lemma, which deals with a class of cones which are not necessarily convex nor proper (notice that in the case of convex proper cones our proof is simpler than that of loc. cit.). This class is wide enough to allow one to deal with various situations, like the case of parameters or the case of complex manifolds.

The author wishes to thank Pierre Schapira for useful discussions during the preparation of this paper.

\section{Review on sheaves}

In this section we recall some notions and results from [5].

1991 Mathematics Subject Classification. 58G99, 18F99. 
Let $X$ be a real $C^{\infty}$ manifold and denote by $D^{b}(X)$ the derived category of the category of bounded complexes of sheaves of $\mathbb{C}$-vector spaces on $X$. With $F \in$ $\mathrm{Ob}\left(D^{b}(X)\right)$, one associates its micro-support $\mathrm{SS}(F)$, a closed conic involutive subset of the cotangent bundle $T^{*} X$. If $\Omega \subset T^{*} X$ is a subset, one denotes by $D^{b}(X ; \Omega)$ the localization of $D^{b}(X)$ with respect to the null system $N=\{F \in$ $\left.\operatorname{Ob}\left(D^{b}(X)\right): \Omega \cap \mathrm{SS}(F)=\emptyset\right\}$.

Let $f: Y \rightarrow X$ be a morphism, and consider the associated correspondence of cotangent bundles:

$$
T^{*} Y \stackrel{t_{f}^{\prime}}{\longleftarrow} Y \times_{X} T^{*} X \stackrel{f_{\pi}}{\longrightarrow} T^{*} X .
$$

It is easy to prove that the micro-support enjoys the following functorial properties:

Lemma 2.1 ([5, Chapter V]). Let $F, F^{\prime} \in \mathrm{Ob}\left(D^{b}(X)\right), G \in \mathrm{Ob}\left(D^{b}(Y)\right)$.

(i) If $f$ is smooth, then $\operatorname{SS}\left(f^{-1} F\right) \subset{ }^{t} f^{\prime} f_{\pi}^{-1}(\operatorname{SS}(F))$.

(ii) If $f$ is proper on $\operatorname{supp}(G)$, then $\operatorname{SS}\left(R f_{*} G\right) \subset f_{\pi}^{t} f^{-1}(\operatorname{SS}(G))$.

(iii) $\mathrm{SS}\left(F \otimes F^{\prime}\right) \subset \mathrm{SS}(F) \times \mathrm{SS}\left(F^{\prime}\right)$.

Without loss of generality, since the problems we will deal with in this paper are of a local nature, from this point onwards we will work on vector spaces.

Let $E$ be a real vector space and consider the map $s: E \times E \rightarrow E$ given by $s(x, y)=x+y$. The convolution $G * F \in \operatorname{Ob}\left(D^{b}(E)\right)$ of $F, G \in \operatorname{Ob}\left(D^{b}(E)\right)$ is defined by

$$
G * F=R s_{!}(G \otimes F) .
$$

Notice that $G * F \simeq F * G$ and $F \simeq \mathbb{C}_{\{0\}} * F \simeq F * \mathbb{C}_{\{0\}}$.

The following claim is an easy consequence of Lemma 2.1.

Lemma 2.2. Let $F, G \in \mathrm{Ob}\left(D^{b}(E)\right)$ and assume that $\operatorname{supp}(F)$ is compact. Then

$$
\mathrm{SS}(G * F) \subset\left\{(x ; \xi) \in T^{*} E: \exists y,(x-y ; \xi) \in \mathrm{SS}(G), \quad(y ; \xi) \in \operatorname{SS}(F)\right\} .
$$

In particular, $\operatorname{supp}(G * F) \subset \operatorname{supp}(G)+\operatorname{supp}(F)$.

Let $D_{\mathbb{R}^{+}}^{b}(E)$ be the full triangulated subcategory of $D^{b}(E)$ whose objects have locally constant cohomology groups along the orbits of $\mathbb{R}^{+}$(the multiplicative group of positive numbers).

Let $H \in \mathrm{Ob}\left(D_{\mathbb{R}^{+}}^{b}\left(E^{*}\right)\right)$. The Fourier-Sato transform of $H$ is the object $H^{\wedge}$ of $D_{\mathbb{R}^{+}}^{b}(E)$ defined by

$$
H^{\wedge}=R q_{1 !}\left(\mathbb{C}_{P} \otimes q_{2}^{-1} H\right),
$$

where we set $P=\left\{(x ; \xi) \in E \times E^{*}:\langle x, \xi\rangle \leq 0\right\}$, and where $q_{1}$ and $q_{2}$ are the natural projections from $E \times E^{*}$ to $E$ and $E^{*}$ respectively. 
In the identification $T^{*} E \simeq E \times E^{*}$, consider the isomorphism

$$
\chi: T^{*} E^{*} \rightarrow T^{*} E, \quad(\xi ; x) \mapsto(x ;-\xi) .
$$

Proposition 2.3 ([5, Theorem 5.5.5]). For $H \in \mathrm{Ob}\left(D_{\mathbb{R}^{+}}^{b}\left(E^{*}\right)\right)$ one has

$$
\mathrm{SS}\left(H^{\wedge}\right)=\chi(\mathrm{SS}(H)) .
$$

Denote by $\omega_{E}$ the dualizing complex of $E$ (recall that $\omega_{E} \simeq \operatorname{or}_{E}[\operatorname{dim} E]$, where $\operatorname{or}_{E}$ denotes the orientation sheaf). Let $\gamma \subset E^{*}$ and $U \subset E$ be locally closed subsets. Assume $\gamma$ conic, and denote by $\gamma^{a}=-\gamma$ its antipodal. Let $F \in \mathrm{Ob}\left(D^{b}(E)\right)$. As a variation of the functor introduced in [5, Exercise V.8], we consider the cut-off functor

$$
\Phi_{U, \gamma}(F)=\left(\mathbb{C}_{\gamma^{a}}^{\wedge} \otimes \omega_{E}\right) * F_{U} .
$$

Notice that if $U$ and $\gamma$ are open, then the natural maps $\mathbb{C}_{U} \rightarrow \mathbb{C}_{E}$ and $\mathbb{C}_{\gamma^{a}} \rightarrow \mathbb{C}_{E^{*}}$, together with the isomorphism $\mathbb{C}_{E^{*}}^{\wedge} \otimes \omega_{E} \simeq \mathbb{C}_{\{0\}}$, induce a natural morphism

$$
\Phi_{U, \gamma}(F) \rightarrow F
$$

Similarly, if $U$ and $\gamma$ are closed, there is a natural morphism

$$
F \rightarrow \Phi_{U, \gamma}(F) .
$$

The following result is a generalization of [5, Proposition 5.2.3].

Proposition 2.4 ([5, Exercise V.8], the microlocal cut-off lemma). Let $U \subset$ $E$ be a locally closed relatively compact subset, let $\gamma \subset E^{*}$ be a locally closed conic subset, and let $F \in \mathrm{Ob}\left(D^{b}(E)\right)$.

(i) The following estimate holds:

$$
\operatorname{SS}\left(\Phi_{U, \gamma}(F)\right) \subset E \times \bar{\gamma} .
$$

(ii) If $U$ and $\gamma$ are open (resp. closed), then the natural morphism (2.4) (resp. (2.5)) is an isomorphism in $D^{b}(E ; \operatorname{Int}(U) \times \operatorname{Int}(\gamma))$.

Since we assumed $U$ to be relatively compact, we can give here a simpler proof than that of loc. cit. (in particular, we will not make use of the " $\gamma$-topology").

Proof. (i) By Proposition 2.3, one has $\operatorname{SS}\left(\mathbb{C}_{\gamma^{a}}^{\wedge} \otimes \omega_{E}\right) \subset\{(x ; \xi): \xi \in \bar{\gamma}\}$. Applying Lemma 2.2 we get $\operatorname{SS}\left(\Phi_{U, \gamma}(F)\right) \subset\{(x ; \xi): \xi \in \bar{\gamma}\}$.

(ii) Assume that $U$ and $\gamma$ are open. The natural morphism (2.4) factors through the morphism $F_{U} \rightarrow F$, and hence it is enough to prove that the morphism $\Phi_{E, \gamma}\left(F_{U}\right) \rightarrow F_{U}$ is an isomorphism in $D^{b}(E ; E \times \gamma)$. One has a distinguished triangle

$$
\Phi_{E, \gamma}\left(F_{U}\right) \rightarrow F_{U} \rightarrow \Phi_{E, E^{*} \backslash \gamma}\left(F_{U}\right) \stackrel{+1}{\longrightarrow},
$$


and (i) implies that

$$
\operatorname{SS}\left(\Phi_{E, E^{*} \backslash \gamma}\left(F_{U}\right)\right) \subset\left\{(x ; \xi): \xi \in E^{*} \backslash \gamma\right\}
$$

The case of $U$ and $\gamma$ closed is similar.

\section{Refined microlocal cut-off}

Let $U$ be a relatively compact neighborhood of 0 in $E$, and let $\gamma$ be a conic open subset of $E^{*}$. In view of Proposition 2.4, it is possible to associate to a sheaf $F \in D^{b}(E)$ a sheaf $\Phi_{U, \gamma}(F)$ which is isomorphic to $F$ in $U \times \gamma$, and whose micro-support is contained in $E \times \bar{\gamma}$.

The problem now is whether it is possible to find pairs $(U, \gamma)$ as above such that the micro-support of $\Phi_{U, \gamma}(F)$ is close to that of $F$ over 0 . For $\gamma$ proper and convex, a positive answer is given by the refined microlocal cut-off lemma of $[5$, Proposition 6.1.4].

Here, we will give the notion of refined cutting pairs $(U, \gamma)$, and prove in Theorem 3.2 below that they satisfy the above requirements. Finally, in order to get examples of refined cutting pairs with $\gamma$ not necessarily convex nor proper, we introduce the notion of polar pairs that will allow us to treat the case of conic neighborhoods of linear subspaces.

Let $E$ be a real vector space. For a subset $S \subset E$, we set $\dot{S}=S \cap \dot{E}$, where $\dot{E}=E \backslash\{0\}$, and if $E^{\prime}$ is another vector space, and $T \subset E \times E^{\prime}$, we also set $\ddot{T}=T \cap\left(\dot{E} \times \dot{E}^{\prime}\right)$. We denote by $S^{\circ}$ the polar set to $S$, defined by

$$
S^{\circ}=\left\{\xi \in E^{*}:\langle x, \xi\rangle \geq 0, \forall x \in S\right\},
$$

and we denote by $N^{*}(S)$ the conormal cone to $S$, as in [5, Definition 5.3.6]. Recall that if $S \subset E$ is an open subset with $C^{1}$-boundary, then $\dot{N}^{*}(S)$ describes the set of interior conormals to $S$. For a subset $\Lambda$ of $T^{*} E$ and $x \in E$, we will use the notation $\Lambda_{x}=\Lambda \cap \pi^{-1}(x)$, where $\pi: T^{*} E \rightarrow E$ is the natural projection.

Let $\gamma \subset E^{*}$ be an open cone. In the identification $T^{*} E \simeq E \times E^{*}$, we set

$$
\partial^{\circ} \gamma=\pi\left(\chi\left(\ddot{\mathrm{SS}}\left(\mathbb{C}_{\gamma}\right)\right)\right)
$$

Notice that if $\gamma$ is an open convex cone, then Proposition 2.3 and [5, Lemma 3.7.10] imply

$$
\partial^{\circ} \gamma=\pi\left(\chi\left(\ddot{\mathrm{SS}}\left(\mathbb{C}_{\gamma}\right)\right)\right)=\pi\left(\ddot{\mathrm{SS}}\left(\mathbb{C}_{\gamma}^{\wedge}\right)\right)=\pi\left(\ddot{\mathrm{SS}}\left(\mathbb{C}_{\gamma^{\circ a}}\right)\right)=\partial \gamma^{\circ a} \backslash\{0\},
$$

where $\partial \gamma^{\circ a}$ denotes the boundary of $\gamma^{\circ a}$. 
Definition 3.1. Let $U \subset E$ be a relatively compact open neighborhood of 0 , and let $\gamma \subset E^{*}$ be an open cone. We say that $(U, \gamma)$ is a refined cutting pair (at $0 \in E$ ) if

$$
\left\{\begin{array}{l}
\text { for any } x \in \partial U \cap \partial^{\circ} \gamma \text { there exists } \xi \in \dot{E}^{*} \text { such that } \\
N_{x}^{*}(U)=\mathbb{R}_{\geq 0} \xi \text { and } \chi\left(\operatorname{SS}\left(\mathbb{C}_{\gamma}\right)\right) \cap \pi^{-1}(x)=\mathbb{R}_{\leq 0} \xi .
\end{array}\right.
$$

Theorem 3.2. Let $(U, \gamma)$ be a refined cutting pair. For $F \in \operatorname{Ob}\left(D^{b}(E)\right)$, one has

$$
\dot{\mathrm{SS}}_{0}\left(\Phi_{U, \gamma}(F)\right) \subset\{\xi \in \gamma:(0 ; \xi) \in \operatorname{SS}(F)\} \cup\{\xi \in \partial \gamma: \exists x \in \bar{U},(x ; \xi) \in \operatorname{SS}(F)\} .
$$

Proof. We will give a proof similar to that of [5, Proposition 5.2.3].

By the microlocal cut-off lemma one only has to prove that

$$
\xi_{\circ} \in \partial \gamma \cap \operatorname{SS}_{0}\left(\Phi_{U, \gamma}(F)\right) \Rightarrow \exists x_{\circ} \in \bar{U},\left(x_{\circ} ; \xi_{\circ}\right) \in \operatorname{SS}(F) .
$$

By Lemma 2.2 and Proposition 2.3, we have

$$
\begin{aligned}
\dot{S S}_{0}\left(\Phi_{U, \gamma}(F)\right) & \subset\left\{\xi \in \dot{E}^{*}: \exists x,(-\xi ;-x) \in \operatorname{SS}\left(\mathbb{C}_{\gamma^{a}}\right),(x ; \xi) \in \operatorname{SS}\left(F_{U}\right)\right\} \\
& =\left\{\xi \in \dot{E}^{*}: \exists x,(\xi ; x) \in \operatorname{SS}\left(\mathbb{C}_{\gamma}\right),(x ; \xi) \in \operatorname{SS}\left(F_{U}\right)\right\},
\end{aligned}
$$

and hence there exists $x_{\circ}$ with

$$
\left(\xi_{\circ} ; x_{\circ}\right) \in \operatorname{SS}\left(\mathbb{C}_{\gamma}\right), \quad\left(x_{\circ} ; \xi_{\circ}\right) \in \operatorname{SS}\left(F_{U}\right) .
$$

In particular, $x_{\circ} \in \partial^{\circ} \gamma \cap \bar{U}$.

If $x_{\circ} \in U$, then $F_{U} \simeq F$ at $\left(x_{\circ} ; \xi_{\circ}\right)$ and hence $\left(x_{\circ} ; \xi_{\circ}\right) \in \operatorname{SS}(F)$.

If $x_{\circ} \in \partial U$, assume by contradiction that $\left(x_{\circ} ; \xi_{\circ}\right) \notin \mathrm{SS}(F)$. Hypothesis (3.2) implies that $N_{x_{\circ}}^{*}(U)=\mathbb{R}_{\geq 0} \xi_{\circ}$. Then Proposition 5.4.8 of [5] gives $\xi_{\circ} \in \mathrm{SS}_{x_{\circ}}\left(F_{U}\right) \subset \mathbb{R}_{\leq 0} \xi_{\circ}+\operatorname{SS}_{x_{\circ}}(F)$, which implies $\left(x_{\circ} ; \xi_{\circ}\right) \in \operatorname{SS}(F)$. This is a contradiction, and this completes the proof.

Let $\gamma \subset E^{*}$ and $\Gamma \subset E$ be open cones. We say that $(\gamma, \Gamma)$ is a polar pair if

$$
\chi\left(\ddot{\mathrm{SS}}\left(\mathbb{C}_{\gamma}\right)\right)=\ddot{\mathrm{SS}}\left(\mathbb{C}_{\Gamma}\right)^{a},
$$

where " $a$ " denotes the antipodal in the fiber variables of $T^{*} E$, i.e. the antipodal map of $E^{*}$. Notice that, if $\gamma \subset E^{*}$ is an open proper convex cone, then $\left(\gamma, \operatorname{Int}\left(\gamma^{\circ a}\right)\right)$ is a polar pair. In fact, one has

$$
\chi\left(\operatorname{SS}\left(\mathbb{C}_{\gamma}\right)\right)=\operatorname{SS}\left(\mathbb{C}_{\gamma}^{\wedge}\right)=\operatorname{SS}\left(\mathbb{C}_{\gamma^{\circ a}}\right)=\operatorname{SS}\left(\mathbb{C}_{\operatorname{Int}\left(\gamma^{\circ a}\right)}\right)^{a} .
$$

As shown in the proof of [5, Proposition 6.1.4], if $\gamma \subset E^{*}$ is an open proper convex cone such that $\partial \gamma^{\circ a} \backslash\{0\}$ is of class $C^{1}$, then there exists a fundamental system $U$ of neighborhoods of $0 \in E$ such that $(U, \gamma)$ is a refined cutting pair. To get other examples of refined cutting pairs, we have the following result. 
Lemma 3.3. Let $(\gamma, \Gamma)$ be a polar pair such that $\partial \Gamma \backslash\{0\}$ is of class $C^{1}$. Then there exists a fundamental system $U$ of neighborhoods of $0 \in E$ such that $(U, \gamma)$ is a refined cutting pair.

Proof. Let us restrict ourselves to considering open subsets $U$ with $C^{1}$ boundary. In this case, assumption (3.2) is equivalent to saying that $\partial U$ and $\partial \Gamma \backslash\{0\}$ are anti-tangent at their intersection, i.e. $N_{x}^{*}(U)=-N_{x}^{*}(\Gamma)$ for each $x \in \partial U \cap \partial \Gamma$.

Denote by $B(0, r)$ and $S(0, r)$ the ball and the sphere of center 0 and radius $r$ in $E$. The idea is then to let $U$ be a smoothing of the set $B(0, r) \cup(B(0,2 r) \backslash \bar{\Gamma})$. To this end, let $(\varrho, \theta)$ be a system of polar coordinates in $E$, and denote by $\operatorname{dist}[\cdot, \cdot]$ the Riemannian distance function on $S(0,1)$. Let

$$
\begin{aligned}
W_{r, \varepsilon}= & \left\{\left(s_{1}, s_{2}\right) \in \mathbb{R}^{2}: s_{1}<r-\varepsilon, s_{2}>0\right\} \cup\left\{\left(s_{1}, s_{2}\right) \in \mathbb{R}^{2}: s_{1}<r, s_{2}>\varepsilon\right\} \\
& \cup\left\{\left(s_{1}, s_{2}\right) \in \mathbb{R}^{2}:\left(s_{1}-r+\varepsilon\right)^{2}+\left(s_{2}-\varepsilon\right)^{2}<\varepsilon^{2}\right\}, \\
M_{r, \varepsilon}= & \left\{\left(s_{1}, s_{2}\right) \in \mathbb{R}^{2}: s_{1}<r\right\} \cup\left\{\left(s_{1}, s_{2}\right) \in \mathbb{R}^{2}: s_{2}<0\right\} \\
& \cup\left\{\left(s_{1}, s_{2}\right) \in \mathbb{R}^{2}: s_{1}<r+\varepsilon, s_{2}<\varepsilon,\left(s_{1}-r-\varepsilon\right)^{2}+\left(s_{2}-\varepsilon\right)^{2}>\varepsilon^{2}\right\},
\end{aligned}
$$

and set

$U=\left\{(\varrho, \theta) \in E:(\varrho, \operatorname{dist}[\theta, S(0,1) \cap \Gamma]) \in W_{2 r, \varepsilon}, \quad(\varrho, \operatorname{dist}[\theta, S(0,1) \backslash \Gamma]) \in M_{r, \varepsilon}\right\}$

For $\varepsilon>0$ small enough with respect to the curvature of $\partial(S(0,1) \cap \Gamma)$, and $r>3 \varepsilon$, it is easily checked that this family of sets $U$ has the desired properties. $\square$

An example of refined microlocal cut-off with respect to a cone not necessarily convex nor proper (a conic neighborhood of a linear subspace) is given by the following proposition.

Corollary 3.4. Let $E$ be a real vector space, let $L$ be a vector subspace of $E$, and let $F \in \mathrm{Ob}\left(D^{b}(E)\right)$. Then there exists a fundamental system $\gamma$ of open conic neighborhoods of $\left(\dot{T}_{L}^{*} E\right)_{0}$ in $T_{0}^{*} E \simeq E^{*}$ such that for each open conic neighborhood $W$ of $\bar{\gamma} \cap \operatorname{SS}(F)$ there exists $F^{\prime} \in \mathrm{Ob}\left(D^{b}(E)\right)$ and a morphism $u: F^{\prime} \rightarrow F$ satisfying the following conditions:

(i) $u$ is an isomorphism in $D^{b}(E ; U \times \gamma)$, for some open neighborhood $U$ of $0 \in E$,

(ii) $\dot{\mathrm{SS}}_{0}\left(F^{\prime}\right) \subset W$.

Proof. The micro-support being a closed set, in view of Proposition 2.4 and Theorem 3.2 it is enough to show that there exists a fundamental system $\gamma$ of open conic neighborhoods of $\left(\dot{T}_{L}^{*} E\right)_{0}$, and for each $\gamma$ there exists a fundamental system $U$ of open neighborhoods of 0 in $E$, such that $(U, \gamma)$ is a refined cutting pair. 
Consider a (non-canonical) decomposition $E=L \oplus\left(T_{L}^{*} E\right)_{0}$, and take $\xi \in$ $\left(\dot{T}_{L}^{*} E\right)_{0}$. Let $\delta$ be a fundamental system of open conic convex neighborhoods of $\xi$ in $L \oplus \mathbb{R} \xi$, such that $\partial \delta \backslash\{0\}$ is of class $C^{1}$. Let $\gamma=\bigcup_{\theta \in \Theta} \theta(\delta)$, where $\Theta$ denotes the family of rotations of $E$ preserving $L$. Then one checks that $(\gamma, \Gamma)$ is a polar pair for $\Gamma=\bigcup_{\theta \in \Theta} \theta\left(\delta^{\circ a}\right)$, where $\delta^{\circ}$ denotes the polar set to $\delta$ in $L \oplus \mathbb{R} \xi$, and one concludes by applying Lemma 3.3.

REMARK 3.5.

(i) In [1], [3], Corollary 3.4 is used to deal with sheaves on complex manifolds. In fact, in this case one is concerned with conic neighborhoods of the complex line generated by a point of the cotangent bundle.

(ii) Theorem 3.2 gives an easier way to get the estimates of the "relative" micro-support, needed in [4].

(iii) We could easily recover the cut-off lemma with parameters of [2, Proposition 2.4.4], where $E=E^{\prime} \times E^{\prime \prime}$, and $\gamma=\gamma^{\prime} \times E^{\prime \prime *}$ for a proper convex cone $\gamma^{\prime} \subset E^{\prime *}$ such that $\dot{\gamma}^{\prime}$ has a $C^{1}$ boundary.

(iv) Of course, it would be possible to state all the results of this section in the case of $U$ and $\gamma$ closed.

\section{REFERENCES}

[1] E. Andronikof, A microlocal version of the Riemann-Hilbert correspondence, Topol. Methods Nonlinear Anal. 4 (1994), 417-425.

[2] A. D'Agnolo, Inverse image for the functor $\mu$ hom, Publ. RIMS Kyoto Univ. 27 (1991), 509-532.

[3] J.-M. Delort, Microlocalisation simultanée et problème de Cauchy ramifié, Compositio Math. 100 (1996), 171-204.

[4] R. IshimuRA, Le foncteur $\mu$ hom relatif et l'action de $\mathcal{E}^{\mathbb{R}}$ relatif sur les fonctions holomorphes, Mem. Fac. Sci. Kyushu Univ. Ser. A 46 (1992), 69-77.

[5] M. Kashiwara And P. Schapira, Sheaves on Manifolds, Grundlehren Math. Wiss., vol. 292, Springer-Verlag, 1990.

Manuscript received March 8, 1995

Andrea D'Agnolo

Institut de Mathématiques

Analyse Algébrique

Université Pierre et Marie Curie

Case 247

4, place Jussieu

F-75252 Paris Cedex 05, FRANCE

E-mail address: dagnolo@mathp6.jussieu.fr

TMNA : Volume $8-1996-\mathrm{N}^{\circ} 1$ 